\begin{tabular}{|c|c|c|c|c|c|c|c|c|c|c|c|c|c|c|c|c|}
\hline 兽 & & & & ol & & & & & & $\begin{array}{c}\mathbf{T} \\
\text { Tem- } \\
\text { peratu! }\end{array}$ & $\begin{array}{c}\text { Dichte bei } \\
\text { neben- } \\
\text { stehender? }\end{array}$ & $\begin{array}{c}\text { Brechings- } \\
\text { exponent } \\
\text { Lei neben- } \\
\text { stehender T }\end{array}$ & $\begin{array}{c}\text { Spezi- } \\
\text { fische } \\
\text { Refraktion }\end{array}$ & $\begin{array}{l}\text { Versei- } \\
\text { fungs- } \\
\text { zahl }\end{array}$ & Jodzahl & $\begin{array}{l}\text { Miitlere } \\
\text { Molekular- } \\
\text { refraktion }\end{array}$ \\
\hline 1 & Leinöl & & & & & & & & . 1 & $18^{\circ}$ & 0,9313 & 1,4800 & 0,5154 & 194,3 & 160,0 & 446 \\
\hline 2 & ", & & . & & 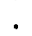 & & . & . & .2 & $18^{\circ}$ & 0,9339 & 1,4810 & 0,5150 & 192,4 & 160,3 & 450 \\
\hline 3 & , & & . & . & & & . & . & .3 & $18^{\circ}$ & 0,9305 & 1,4795 & 0,5155 & 193 & 161,1 & 448 \\
\hline 4 & & & . & . & 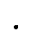 & & & . & . 4 & $18^{\circ}$ & 0,9321 & 1,4800 & 0,5149 & 194,0 & 153,3 & 446 \\
\hline 5 & & & . & . & . & & . & . & . 5 & $18^{\circ}$ & 0,9336 & 1,4800 & 0,5141 & 193,5 & 150,4 & 446 \\
\hline 6 & & & . & & & & & & .6 & $22,5^{\circ}$ & 0,9290 & 1,4800 & 0,5167 & 194,7 & 150,4 & 446 \\
\hline 7 & Cottonöl & & & & & & 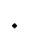 & . & .1 & $23^{\circ}$ & 0,9215 & 1,4730 & 0,5133 & 198,9 & 108,7 & 434 \\
\hline 8 & ," & & 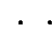 & & & & & & .2 & $19^{\circ}$ & 0,9186 & 1,4710 & 0,5127 & 197,0 & 101,7 & 437 \\
\hline 9 & & & 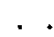 & 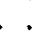 & & & 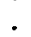 & & . 3 & $19^{\circ}$ & 0,9196 & 1,4715 & 0,5127 & 196,5 & 109,3 & 438 \\
\hline 10 & Rüböl & & . & . & 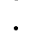 & & . & . & . 1 & $18^{\circ}$ & 0,9169 & 1,4735 & 0,5164 & 172,8 & 99,8 & $502-503$ \\
\hline 11 & ", & & . & 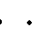 & & . & . & & .2 & $18^{\circ}$ & 0,9179 & 1,4735 & 0,5159 & 176,8 & 105,5 & $490-492$ \\
\hline 12 & ", & & . & & . & . & . & . & . 3 & $18^{\circ}$ & 0,9058 & 1,4725 & 0,5216 & 174,1 & 99,1 & 503 \\
\hline 13 & , & & . & . & . & . & . & . & . 4 & $18^{\circ}$ & 0,9131 & 1,4735 & 0,5186 & 175,4 & 98 & 491 \\
\hline 14 & & & . & . & . & 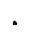 & . & . & .5 & $18^{\circ}$ & 0,9128 & 1,4710 & 0,5 & 17 & 93,3 & 488 \\
\hline 15 & Hederichö & & . & & & & & & . & $18^{\circ}$ & 0,9236 & 1,4730 & & & 107 & 487 \\
\hline 16 & Maisöl. & & . & • & . & . & . & & . . & $23^{\circ}$ & 0,9234 & 1,4 & & & 117 & 448 \\
\hline 17 & Olivenöl. & & & & & & . & 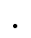 & . 1 & $15^{\circ}$ & 0,9 & & & & & 9 \\
\hline 18 & & & & . & & & & . & .2 & $15^{\circ}$ & 0,9 & & & & & 7 \\
\hline 19 & & & . & & & & & & 3 & $15^{\circ}$ & 0,91 & & & & & 4 \\
\hline 20 & & & & & & & & & . 4 & $15^{\circ}$ & 0,9121 & 1,4 & & & & 4 \\
\hline 21 & & & & & & & & & 5 & $15^{\circ}$ & 0,9145 & 1,46 & & & & 4 \\
\hline 22 & Sojaöl & & & & & & & & 1 & $15^{\circ}$ & 0,9235 & 1,4731 & 0,5124 & 192,2 & 125,3 & 447 \\
\hline 23 & & & & & & & & & 2 & $15^{\circ}$ & 0,9240 & 1,4732 & $0,512 \mathrm{l}$ & 191,7 & 125,0 & 449 \\
\hline 24 & & & & & & & & & 3 & $15^{\circ}$ & 0,9264 & 1,4741 & 0,5118 & & 130 & $4 t$ \\
\hline 25 & & & & & & & & & 4 & $15^{\circ}$ & 0,9230 & 1,4 & & & & 451 \\
\hline 26 & & & & & & & & & 5 & $15^{\circ}$ & 0,9242 & 1,4 & & & & 44 \\
\hline 27 & & & & & & & & & 6 & $15^{\circ}$ & 0,9231 & 1,4745 & 40 & 2,2 & 13 & 449 \\
\hline 28 & Sojä̈l mi & & Cott & toni & & & & & 7 & $15^{\circ}$ & 0,9270 & 1,4738 & 0,5111 & 191,6 & 132,5 & 448 \\
\hline 29 & , & & & & & & & & . 8 & $15^{\circ}$ & 0,9287 & 1,4745 & 0,5109 & 192,0 & 134,2 & 448 \\
\hline 30 & & & & & & & & & 9 & $15^{\circ}$ & 0,9240 & 1,4740 & 0,5129 & 192,6 & 135,6 & 448 \\
\hline 31 & Klauenöl & & & & & & . & & . & $15^{\circ}$ & 0,9177 & 1,4679 & 0,5099 & 185,3 & 82,8 & 462 \\
\hline 32 & Sesamöl & & & & & . & • & & . 1 & $23^{\circ}$ & 0,9195 & 1,4730 & 0,5144 & 192,6 & - & 449 \\
\hline 33 & ," & & & . & 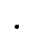 & - & & & & $23^{\circ}$ & 0,9189 & 11,4725 & 5142 & 189,4 & - & 456 \\
\hline 34 & 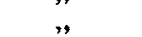 & & & & & . & & & . 3 & $23^{\circ}$ & 0,9185 & & & & - & 448 \\
\hline 35 & 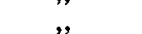 & & & & & & & & & $23^{\circ}$ & 0,9192 & & & 192,1 & - & \\
\hline 36 & $"$ & & & & & & & & .5 & $23^{\circ}$ & 0,9195 & 1,4730 & & 192,6 & - & 449 \\
\hline 37 & $"$ & & & & & & & & .6 & $23^{\circ}$ & 0,9193 & 1,4725 & & & -- & 4 \\
\hline 38 & & & & & & & & & & $18^{\circ}$ & 0,9234 & 1,4730 & 0,5122 & 188,3 & 103,9 & 457 \\
\hline 39 & Erdnußöl & & & & & & & & 1 & $22^{\circ}$ & 0,9139 & 1,4700 & 0,5143 & & - & 451 \\
\hline 40 & 20.6- & & & & & & & & & $22^{\circ}$ & 0,9328 & 1,4750 & 0,5092 & 192,3 & 99,9 & 445 \\
\hline
\end{tabular}

III. Sesa möl. 4.

$$
\begin{aligned}
& \begin{array}{c}
\mathrm{D}(\mathbf{P})^{23^{\circ}}==0,9192 \quad \mathrm{n}^{23^{\circ}}=1,4725 \quad \mathrm{~V}==192,1 \\
\mathrm{M}=874,5
\end{array}
\end{aligned}
$$

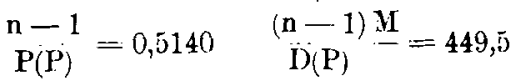

$$
\begin{aligned}
& \mathrm{D}(\mathbf{P})^{28^{\circ}}=0,9192 \quad \mathbf{n}^{16^{\circ}}=1,4730 \\
& \frac{\mathrm{n}-1}{\mathrm{D}(\mathrm{P})}=0,5146 \quad \frac{(\mathrm{n}-1) \mathbf{M}}{\mathrm{D}(\overline{\mathrm{A}})}=450,0 \text {. }
\end{aligned}
$$

Das Beispiel III erweist, daß ein Unterschied von wenigen Graden in Temperaturen, bei welchen das Brechungsvermögen und die Dichte festgestellt werden, nicht von so bedeutendem Einflusse ist, daß dio spezifische Refraktion oder die mittlere Molekularrefraktion sich wesentlich ändern. - Es genügt daher, will man letzteren Wert feststellen, die Zimmertemperatur zu wählen.

In der folgenden Tabelle sind die Werte der mittleren Molekularrefraktion auf Einheiten abgerundet. Der Brechungsindex ist bei weißem Tageslichte in einem $\mathrm{Z} \mathrm{e} \mathrm{i} \mathrm{B} \mathrm{schen} \mathrm{Apparate} \mathrm{fest-}$ gestellt. Auffallend sind die hohen Verseifungszahlen bei amerikanischen Baumwollsamenölen und die niedrigen Verseifungszahlen bei zwei, Sesam. ölen, deren Provenienz nicht - festgestellt werden konnte, und deren Echtheit nicht zweifellos ist. Die Zahlen wurden, um Zufälligkeiten auszuschalten, durch Wiederholungsversuche kontrolliert. Die Daten von 17 Ölen hat mit gütiger Erlaubnis des Herrn Hofrathes Dr. F, D a f e t mir die K. K. landwirtschaftlich-chemische Versuchsstation in Wien zur Verfügung gestellt, wofür ich hiermit bestens danke.

\section{Über die Einwirkung von Wasser und Alkali auf Baumwollcellulose.}

Von Carl G. Sch walbe und Michael Robivofy.

(Eingeg. 4./1. 1911.)

\section{Vorläufige Mitteilung. $\left.{ }^{1}\right)$}

$T$ a $u$ B 2) hat im Jahre 1889 behauptet, daß Cellulose beim Erhitzen mit Wasser auf hohe Tem-

1) Bei eingehenderem Studium der in der Oberschrift genannten Reaktion wurde eine Reihe allgemeiner interessanter Beobachtungen gemacht, die im folgenden schon vorweg mitgeteilt werden 
peraturen in Hydrocellulose übergehe. Fine Nachprïfung der $\mathrm{T}$ a $\mathrm{u} B \mathrm{~s}$ c h e $\mathrm{n}$ Angaben scheint bisher nicht erfolgt zu sein. Wir fanden, daß die Bildung von Hydrocellulose, d. h. stark reduzierender Sub. stanz nur dann eintritt, wenn man, wie $T$ a $u B$ es getan, eine schon etwas chemisch veränderte Cellulose, nämlich Filtrierpapier oder eine zu stark ge- bleichte Cellulose anwendet. Geht man aber von völlig reiner Cellulose aus, die weder Druckkochung, noch starke Bleiche durchgemacht hat, so ist die Hydrolysierung selbst bei $20 \mathrm{Atm}$. Druck nur gering. Uber $150^{\circ}$ wird die Zersetzung der Cellulose merklich; man sollte also höhere Temperaturen als $150^{\circ}$ nach Möglichkeit vermeiden.

I a be lle $\mathrm{I}$.

\begin{tabular}{|c|c|c|c|c|c|c|c|c|c|c|}
\hline $\begin{array}{l}\text { Ausgangs- } \\
\text { inaterial }\end{array}$ & $\begin{array}{l}\text { Temperatur } \\
\text { des Wassers }\end{array}$ & ca. $20^{\circ}$ & $100^{\circ}$ & $105^{\circ}$ & $120^{\circ}$ & $135^{\circ}$ & $150^{\circ}$ & $165^{\circ}$ & $179^{\circ}$ & $213^{\bullet}$ \\
\hline $\begin{array}{l}\text { Cellulose } \\
\text { rein }\end{array}$ & $\begin{array}{l}\text { Korrigierte } \\
\text { Kupferzahl }\end{array}$ & 0,042 & 0,109 & 0,114 & 0,127 & 0,153 & 0,19 & 0,26 & 0,30 & - \\
\hline $\begin{array}{l}\text { Cellulose bei } \\
\text { der Bleiche } \\
\text { beschädigt }\end{array}$ & do. & 0,368 & 0,312 & - & 0,331 & 0,40 & 0,479 & 1,43 & 1,78 & 3,43 \\
\hline
\end{tabular}

Um eine reine Baumwollcellulose zu erhalten, die durch Druckkochung noch nicht gelitten hat, sind wir von einem sehr reinen, ungebleichten MakoKardenband ausgegangen, haben es nach einer Vorschrift von 'I a $\mathrm{m}^{\text {i }} \mathrm{n}^{3}$ ) ohne Druck mit Harzseifen-Alkalilösung abgekocht, heiß gewaschen und sehs vorsichtig gebleicht. Wir haben so ein Material mit der korrigierten „Kupferzahl“4) 0,04 herstellen können.

Bei der Bleiche mit Hypochloritlösung nebst nachfolgendem ,Absäuern" mit Salzsäure (oder Essigsäure) hat sich das bemerkenswerte Resultat ergeben, daß dïnnere Säure eine Erhöhung der Kupferzahl, also Oxycellulosenbildung hervorruft. Bei einer Säurekonzentration von $0,1 \%$ war z. $B$. die Kupferzahl 0,15, bei $0,01 \%$ ergab sich 0,28Allerdings war das Weiß bei der geringeren Säure. konzentration noch reiner.

Die Einwirkung von kaltem Alkali auf Baumwollcellulose für Alkalikonzentrationen von 15 bis $30 \%$ (Mercerisation) ist in den letzten Jahren sehr eingehend studjert worden. Dagegen fehlt es an Zahlenmaterial iiber die Finwirkung dünnerer Alkalilaugen.

Wir fanden, daß kalte Laugen die Baumwollcellulosen am stärksten in der Konzentration von $4 \%$ angreifen; insofern als die korrigierte Kupferzahl bei dieser Konzentration ihren Höchstwert erreicht. (Tabelle II.)

Tabelle II.

\begin{tabular}{|c|c|c|c|c|c|c|c|c|c|}
\hline $\begin{array}{l}\text { Ausgangs- } \\
\text { matèrlal }\end{array}$ & $\left\{\begin{array}{c}\text { Prozentgehalt } \\
\text { der I Iauge }\end{array}\right.$ & Wasser & $1 \%$ & $2 \%$ & $3 \%$ & $4^{0} \%$ & $5 \%$ & $6 \%$ & $7 \%$ \\
\hline $\begin{array}{l}\text { Cellulose } \\
\text { rein }\end{array}$ & $\left\{\begin{array}{l}\text { Korrigierte } \\
\text { Kupferzahl }\end{array}\right.$ & 0,04 & 0,150 & 0,166 & 0,195 & 0,257 & 0,135 & 0,142 & 0,154 \\
\hline
\end{tabular}

Dagegen sinkt die Löslichkeit der Bąumwolle stetig mit zunehmencler Konzentration (von 1-8\%) Bei $\mathbf{n}$ und $10 \%$ iger Lange läßt sich die Löslichkeit nicht mehr bestimmen; es tritt eine Crewichts- vermehrung ein, ïber deren Ursachen vorderhand bis zum Abschluß der Untersuchungen eine Hypothese noch nicht aufgestellt werden soll. (Tabelle III.)

Ta belle III.

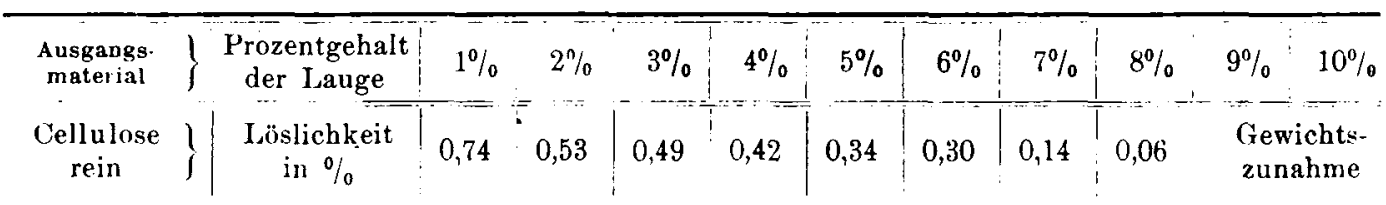

Bei lı̈heren Temperaturen ( $100^{\circ}$ und mehr) steigt die Iöslichkeit, sinkt aber mit zunehmender Konzentration. Die Jöslichkeit wird recht beträchtfich bei Temperaturen über $150^{\circ}$.

sollen, weil sich die Vollendung der geplanten Untersuchung noch einige Zeit hinziehen wird, und wir uns ungestörte Weiterarbeit sichern möchten.

2) Dingl. Journ. 273, 276-285 (1889); 276, $411--428$ (1890).

3) T a m i n, Rev, mat. col. 12, 313-320 (1908)

4) S c h w a l be, diese Z. 23, 924-928 (1910).
Neben der Iöslichkeit ist auch die Gummizahl bestimmt worden, d. h. das Gewicht der durch Alkohol nach dem Neutralisieren alkalischer Auszüge ausfallenden amorphen Flocken. Bei dem völlig reinen Baumwollmaterial konnten Gummizahlen überhaupt nicht erhalten werden, wohl aber bei weniger reinem, durch Bleiche usw, angegriffenem Material. Bei $150^{\circ}$ ergaben sich nur geringe Unterschiede. Der Wert dieser Gummizahlen wird stark beeinträchtigt durch den hohen Kochsalzgehalt. Es reißen die kolloiden Flocken Kochsalz mit hernieder. t'ber $150^{\circ}$ ergeken sich beträchtliche Werte für die 
Gummizall. Auch hier erscheint die Temperatur von $150^{\circ}$ wieder als eine ,,kritische Temperatur" der Baumwollcellulose.

Werden die Kupferzahlen der mit he i $\beta$ en Laugen behandelten Raumwollcellulosen festgestellt, so ergibt sich -- wie bei der kalten Lange - die Konzentration von $4_{\%}^{\circ}$ als besonders verderblich ${ }^{5}$ ) für Baumwollcellulose zu erkennen; $3 \%$ und $5 \%$ sind weit unschädlicher. ('Tabelle IV.)

Tabelle IV.

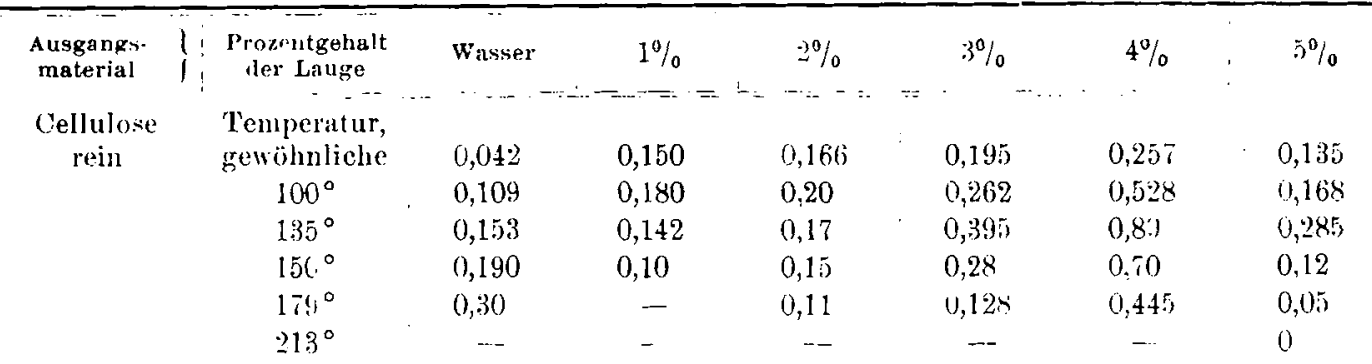

Die durch 1 - und $2 \%$ ige Natronlauge entstehenden Hydrolysenprodukte scheinen oberhalb $100^{\circ}$ schon wieder einer Zersetzung zu unterliegen, denn die Kupferzahlen nehmen ab; bei den Konzentrationen von $3 \%$ und mehr tritt diese Abnahme (Kersetzung) erst oberhalb $135^{\circ}$ auf.

Vielleicht ist die Abnahme der Hydrolyse bei Konzentrationon ron $5 \%$ und mehr auf Rechnung beginnender Mercerisation zu setzen. Wälrend noch Lauge von $4^{\circ} \%$ stark hydrolysiert, tritt bei der Lauge von 5\% die Hydrolyse zugunsten der Hydratisierung (Mercerisation) zurïck.

Aus den bisherigen Versuchen ergibt sich für dic Praxis, daß nın beim Bäuchen der Baumwolle Temferaturen über $150^{\circ}$ und die kritische Laugenkonzentration von $4^{\circ}$; vermeiden soll. Bein Bleichen sind zum Absäuem Säurekonzentrationen von $0,1^{\circ}$ ausreichend: ja mit niedrigerer Konzentration wird das Weiß noch besser, allerdings unter gewisser Hihöhung des (Ixycellulosengehaltes.

Wir setzen die Untersuchungen in Richtung in uf die Hydrolysiergeschwindigkeit, und die damit in Beziehung stehenden Hydratisierung fort; anch die chenischen Veränderungen der Baumwollcellulose bei vollständiger und unvollständiger Mercerisation sollen noch näher studiert werden: nicht nur in An" endurg auf Mako-Baumwolle, sondern auch andere Baumwollrassen. Fs scheint z. B. amerikanische Baumwolle weit höhere Kupferzahlen zu haben als izgytische. Wir beabsichtigen, auch die Veränderungen zu studieren, die Baumuollcellulose hei t e chn is ch e r...Bäuche" mit verschiedenen Alkalikonzentrationen erleidet.

Darmstadt.

Institut für organische Chemie der Technischen Hochschule.

\section{Flïssigkeitsstandmesser.}

Von J r. Ing. Richand.

(Eingeg. 31.12. 1910.)

Recht häufigr steht man in chemischen $\mathrm{Be}$ trieben ror der Aufgabe, eine Reihe von Bottichen, offene und geschlosisene, unter Druck und Vakuum befindliche Kessel auf ihren Flïssigkeitsstand prüfen zu müssen. sei es nun. um einen Bestand aufzunehmen, oder um Mischungen versehiedenw? Flüssigkeitsmengen in einem Kessel vorzunehmen usw. Das Anbringen eines Wasserstandrohrs, welches seitliche Durchbohrungen des Kessels voran-setzt, ist oft unmöglich, oft mit Betriebsechwieriykeiten verknüpft. I) hilft man sich in allgemeinen dureh Abstechen der Flïssigkeitsstände mit Meßstäuen. MeBröhren usw. Joiese Messungen sind hïufig umbequem, zeitraubend und fehlerhaft. entweder der MeBstab, wird nicht ganz vertikal in die Flïssigrkeit getaucht oder die Benetzungsstelle ist nicht fettfrei. und die Flüssigkeit adhäriert nicht an der richtigen Stelle oder aber die Flüssigkeitsoberfläche ist mit einer Sichicht von Schmutz oder Sohaum bedeckt. Wenn endlich mehrere Flüssigkeitsstände in kurzer Zeit - bei der Aufnahme eines Bestands - festgestellt werden sollen. so hat man fiir gründliche Reinigung der Meßstäbe Sorge zl1 tragen. Ias ist namentlich, wenn saure und ätzende Flüssigkeiten zu messen sind, keine angenehme Aufgabe. Ätzungen der Haut und Beschädigung der Kleider sind häufige Begleiter-cheinungen.

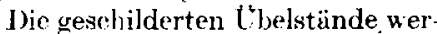
den vermieden. wenn man einen Flüs-

sigkeitsmesser besitzt, welcher belielng tïr rerschiedene Pottiche oder Kessel verwendhar ist, und welcher ebenso als ständiger wie als periodischer Flüssigkeitsstandmesser zu verwerten ist.

Die im folgenden kurz beschriebene und veranschaulichte Vorrichtung soll diesem Zweck dienen; sie ist gesetzlich geschützt und wird ron der Firma Franz llugershoff, Leipzig, Karolinenstraße 13. hergestellt. Sie besteht im wesentlichen aus einen Tauchrohr a (Abb). 1), welehes mit einem

5) Es ist in ditser Hinsicht auch bemerkenswert, daß S c he u rer (Bull. Mulhouse 58, 3(i: [1888] fesigestellt hat, da $3 \%$ ige Iauge beim Bäuchen die Baumwolle stärker in der Festigkeit zurückgehen läßt als 2 - und $8_{0}^{\circ}$ ige Lauge. 